\title{
ARTICLE Replicating predictive serum correlates of greater translocator protein distribution volume in brain
}

Sophia Attwells ${ }^{1,2}$, Elaine Setiawan ${ }^{1}$, Alan A. Wilson ${ }^{1,3}$, Pablo M. Rusjan ${ }^{1}$, Laura Miler ${ }^{1}$, Cynthia Xu ${ }^{1}$, Celeste Hutton ${ }^{1}$, Muhammad I. Husain ${ }^{3}$, Stephen Kish ${ }^{1,2,3}$, Neil Vasdev ${ }^{1}$, Sylvain Houle ${ }^{1,3}$ and Jeffrey H. Meyer ${ }^{1,2,3}$

Greater activation of glia, a key component of neuroinflammation, is an important process to target in neuropsychiatric illnesses. However, the magnitude of gliosis varies across cases so low-cost predictors are needed to stratify subjects for clinical trials. Here, several such blood serum measures were assessed in relation to TSPO $\mathrm{V}_{\mathrm{T}}$, an index of translocator protein density, measured with positron emission tomography. Blood serum concentration of several products known to be synthesized by activated microglia (and to some extent astroglia) [prostaglandin $\mathrm{E}_{2}\left(\mathrm{PGE}_{2}\right)$, prostaglandin $\mathrm{F}_{2}$ alpha $\left(\mathrm{PGF}_{2 \mathrm{a}}\right)$, and tumor necrosis factor alpha $\left(\mathrm{TNF}_{\mathrm{a}}\right)$ ], controlled by an index of peripheral inflammation [C-reactive protein (CRP)] and TSPO $\mathrm{V}_{\mathrm{T}}$ were measured in 3 cohorts: prefrontal cortex TSPO $\mathrm{V}_{\mathrm{T}}$ of 20 subjects with major depressive episodes (MDEs) from major depressive disorder (MDD); and 56 subjects with treatment resistant MDEs from MDD; and dorsal caudate TSPO $\mathrm{V}_{\mathrm{T}}$ of 20 subjects with obsessive-compulsive disorder. Ln(PGE $\left.2 / \mathrm{CRP}\right)$ and $\ln \left(\mathrm{TNF}_{\alpha} / \mathrm{CRP}\right)$ consistently correlated with TSPO $\mathrm{V}_{\mathrm{T}}\left(R^{2}=0.36\right.$ to $0.11, p=0.0030$ to $\left.p=0.0076\right)$. Assessment of threshold serum values to predict highly elevated TSPO $V_{T}$, demonstrated that a positive predictive value (PPV) of $80 \%$ was possible while retaining $40 \%$ of participant samples and that receiver operating curves (ROC) ranged from 75 to $81 \%$. Post-hoc selection of In(CRP) was more predictive $\left(R^{2}=0.23\right.$ to $0.39, p=0.0058$ to $p=0.00013$; ROC $\left.>80 \%\right)$. Systematic assessment of selected peripheral inflammatory markers is promising for developing low cost predictors of TSPO $\mathrm{V}_{\mathrm{T}}$. Marker thresholds with high PPV will improve subject stratification for clinical trials of glial targeting therapeutics.

Neuropsychopharmacology (2020) 45:925-931; https://doi.org/10.1038/s41386-019-0561-y

\section{INTRODUCTION}

Psychiatric and neurological diseases are burdensome to society since they affect one in four people and are often treatment resistant [1]. Neuroinflammation, usually in response to neuronal damage is an important cluster of processes that occurs across many neuropsychiatric illnesses including major depressive disorder (MDD), obsessive-compulsive disorder (OCD), and neurodegenerative diseases [2-8]. Such responses typically include microglial and/or astroglial activation, which involve morphological changes of an enlarged cell body and thickened dendrites or, for microglia, possibly an ameboid shape. Increased gliosis is a promising target for immune modulating treatments to alter microglial and/or astroglial function away from potentially harmful roles like producing reactive oxygen species, prostaglandins, and proteinases. Immune modulating treatments may also alter glia cells towards more curative roles such as enhancing release of neurotrophic factors, promoting vascularization, and phagocytosing cellular debris $[6,9]$. However, a critical barrier for human clinical trials of investigational treatments targeting gliosis is that there is heterogeneity in the neuroinflammatory response among individuals with neuropsychiatric diseases. Heterogeneity is attributable to multiple factors including stage of illness, comorbid disease and, most likely, multiple etiological phenotypes $[2,3]$; thereby limiting optimal matching of cases to treatment.
No easily applicable, replicable, low cost measure indicative of microglial activation has been developed. Presently positron emission tomography (PET) imaging of translocator protein (TSPO) binding, is the most established in vivo marker although there are nuances for its interpretation: In health, binding of TSPO in brain is considered mainly attributable to binding to endothelial cells [10]. After inflammatory stimuli, brain TSPO binding is elevated and this TSPO radiotracer binding closely parallels the magnitude of greater TSPO expression in microglia, with a modest contribution from greater TSPO expression in activated astrocytes [11, 12] (for further discussion see Supplementary Information). Other PET radiotracers targeting $\mathrm{P} 2 \mathrm{X} 7, \mathrm{P} 2 \mathrm{Y} 12$ and $\mathrm{CSF} 1$ receptors $[13,14]$ are being advanced, but all of these methods are expensive, require scarce resources, and typically need arterial blood sampling for kinetic modeling because microglia are ubiquitous throughout the brain. Another direction is cerebrospinal fluid concentration of products consequent to greater indoleamine 2,3-dioxygenase activity and/or cytokines but the necessary lumbar puncture is difficult for many patients $[15,16]$. In regards to magnetic resonance imaging (MRI) methods with paramagnetic probes like Cd-bis-5-HT-DTPA that measure myeloperoxidase activity across activated microglia, neutrophils and monocytes, their sensitivity to detect microglial activation in neuropsychiatric disease is not yet established and it is possible that their application will be restricted to disease states

\footnotetext{
${ }^{1}$ Research Imaging Centre and Campbell Family Mental Health Research Institute at the Centre for Addiction and Mental Health, 250 College Street, Toronto, ON M5T 1R8,

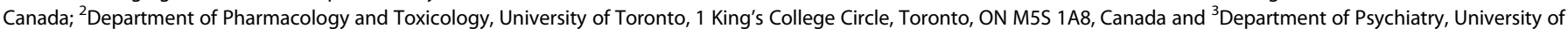
Toronto, 250 College Street, Toronto, ON M5T 1R8, Canada Correspondence: Jeffrey H. Meyer (jeff.meyer@camhpet.ca)
}

Received: 25 July 2019 Revised: 24 September 2019 Accepted: 11 October 2019 Published online: 4 November 2019 
with substantial blood brain barrier breakdown [17]. Moreover, to date, no blood marker has been demonstrated to consistently predict the level of microglial activation in brain.

For the present study, to design low cost blood markers, composite measures were created. Activated microglia in brain were considered an important source of inflammatory markers. Several products of activated microglia that are also actively transported out of the central nervous system into blood were measured, including serum prostaglandin $\mathrm{E}_{2}\left(\mathrm{PGE}_{2}\right)$, prostaglandin $F_{2}$ alpha $\left(\mathrm{PGF}_{2 \mathrm{a}}\right)$, and tumor necrosis factor alpha $\left(\mathrm{TNF}_{\mathrm{a}}\right)$ [18-22]. $\mathrm{PGE}_{2}$ was given the highest priority since it is arguably the most commonly measured product of activated microglia among in vitro studies [18]. Since $\mathrm{PGE}_{2}, \mathrm{PGF}_{2 \alpha}$, and $\mathrm{TNF}_{\alpha}$ are also produced by the immune response of peripheral tissues [23] and microglial activation in brain is influenced by peripheral inflammation [9], a controlling measure of a well-accepted index of peripheral inflammation, serum C-reactive protein (CRP) concentration was applied. Hence, our primary hypothesis is that $P G E_{2} / C R P$ will predict TSPO total distribution volume $\left(\mathrm{V}_{\mathrm{T}}\right)$ in brain and $\mathrm{PGF}_{2 \mathrm{a}} / \mathrm{CRP}$ and $\mathrm{TNF}_{\mathrm{a}} / \mathrm{CRP}$ are our secondary candidates. While the issue could be raised that during inflammatory states astrogliosis may also contribute to the measure of TSPO $V_{T}$, to some extent this issue is mitigated because $\mathrm{PGE}_{2}, \mathrm{PGF}_{2 a}$ and $\mathrm{TNF}_{\alpha}$ are not selective products of microglial activation and are also produced by astrogliosis.

In the present study, the relationship of these serum markers to brain TSPO $V_{T}$ is assessed in three groups: medication free MDD; antidepressant treated, treatment resistant MDD (TRD); and medication free OCD subjects, illnesses for which TSPO $V_{T}$ is elevated yet there is also substantial variability across subjects [2-8] (for further review see the Supplementary Information). In medication free MDD and TRD we prioritized the prefrontal cortex (PFC) because subregions of the PFC are often adversely affected in these illnesses [24-26], and it is a large portion of brain tissue. In OCD, the caudate nucleus was prioritized because it is the region with the most abnormally elevated TSPO $V_{T}$ [2], and is strongly implicated in the pathophysiology of OCD, having the greatest convergence of neurochemical abnormalities across investigations in OCD [27-29].

\section{MATERIALS AND METHODS}

\section{Participants}

Three cohorts were recruited to assess the relationship between PET and peripheral markers. Having three cohorts provides the ability to assess the generalizability of this relationship across these disease conditions and the extent to which it replicates across these disease conditions. The cohorts included: 20 with medication free MDE [7]; 56 TRD (31 previously described [3]); and 20 medication free OCD (19 previously described [2]) were recruited from the Greater Toronto Area and the Centre for Addiction and Mental Health between July 2010 and October 2018 (Table 1). To assess the diagnosis, all participants underwent the Structured Clinical Interview for DSM-IV with confirmation by a consultation with a psychiatrist (JHM). All participants were currently experiencing significant symptoms. For MDE and TRD participants, a minimum of 17 on the 17-item Hamilton Depressive Rating Scale (17-item HDRS) [30] was required because this is a commonly applied minimum threshold to verify a current MDE. Greater TSPO $\mathrm{V}_{\mathrm{T}}$ is commonly found during an MDE, but this may not necessarily occur during recovery $[5,8]$. For OCD participants, scores on the Yale-Brown Obsessive-Compulsive Scale (Y-BOCS) [31] reflected moderate to severe illness. All participants were aged 18-72 years, non-cigarette smoking, and otherwise in good physical health (Table 1). All TRD participants were taking at least one antidepressant medication at a standard clinical dose for a minimum of 4 weeks prior to PET scanning (detailed list of medications provided in Supplementary Information).

All participants provided written informed consent after all procedures were fully explained. The study, protocol, and informed consent forms were approved by the Research Ethics Board at the Centre for Addiction and Mental Health.

Image acquisition and analysis

As previously described $[2,3,7]$, each participant underwent one $\left[{ }^{18}\right.$ F]FEPPA PET (HRRT; CPS/Siemens, Knoxville, TN, USA) and one MRI scan at the Research Imaging Centre at the Centre for Addiction and Mental Health (see the online Supplementary Information for additional detail). $\left[{ }^{18}\right.$ F]FEPPA was administered

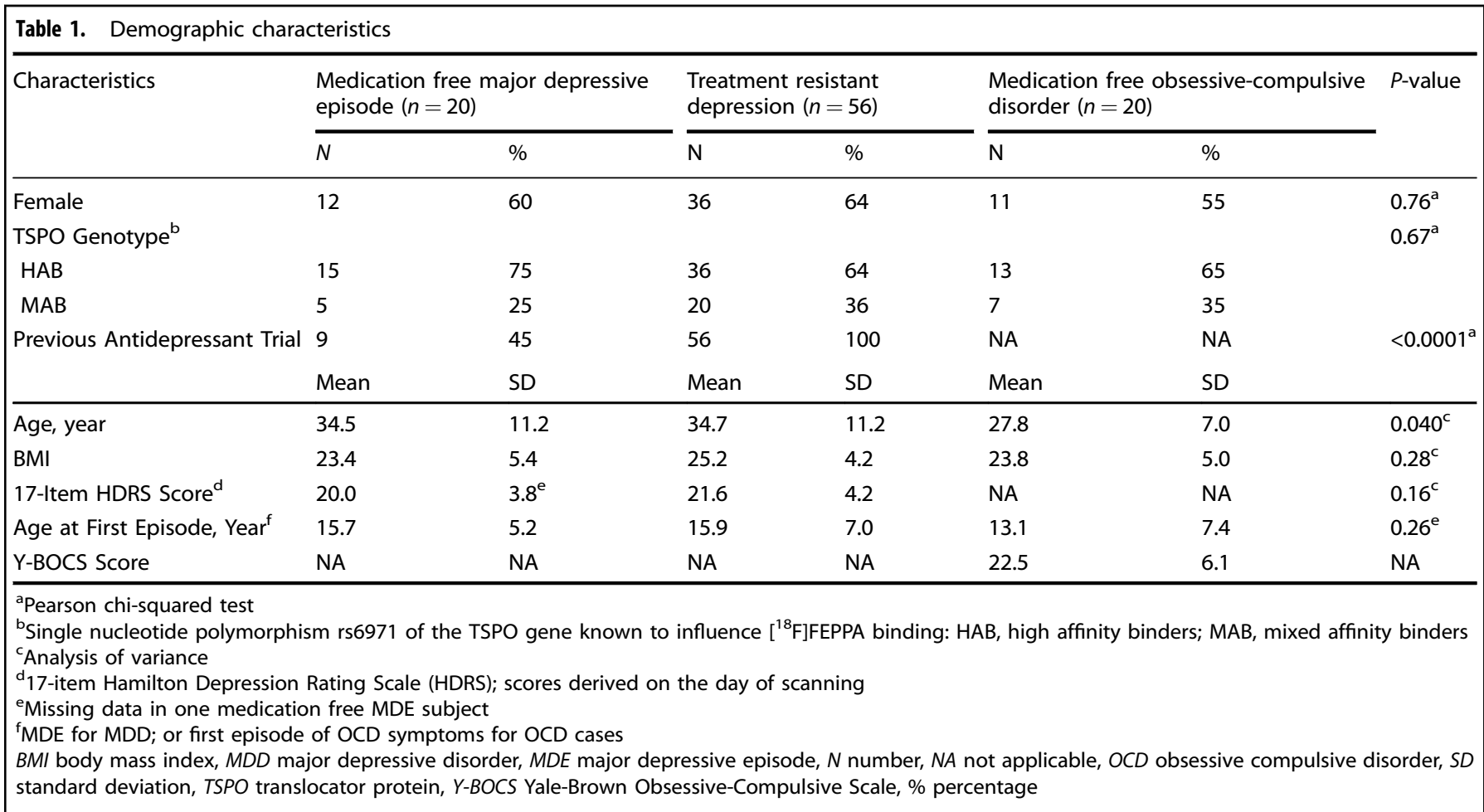


intravenously as a bolus (mean $184.6 \mathrm{MBq}$ [SD 13.0]). $\left[{ }^{18} \mathrm{~F}\right] \mathrm{FEPPA}$ was of high radiochemical purity (>96\%) and high specific activity (mean 83.1 TBq/mmol [SD 73.0]). Manual and automatic blood samplings (ABSS, Model \#PBS-101; Veenstra Instuments, Joure, The Netherlands) were obtained to determine the unmetabolised parent radioligand in plasma which was the input function for the kinetic analysis. A brain MRI was acquired for each participant for the anatomical delineation of regions of interest (ROIs) generated using the semi-automated software (ROMI, Toronto, Ontario) [32]. A two-tissue compartment model was applied to the time-activity curves from regions of interest to measure TSPO $\mathrm{V}_{\mathrm{T}}$, which is the optimal model for $\left[{ }^{18} \mathrm{~F}\right] \mathrm{FEPPA}$ PET [33] (see the online Supplementary Information for additional detail).

Peripheral inflammatory marker measurements

$\mathrm{PGE}_{2}$ and $\mathrm{PGF}_{2 a}$ concentrations were determined using the competitive immunoassay, Prostaglandin $E_{2}$ Assay (Parameter, $R \& D$ Systems Inc) and Prostaglandin $F_{2 a}$ ELISA Kit (MyBioSource), respectively. $\mathrm{TNF}_{\mathrm{a}}$ level was analyzed using the Human Adipokine Magnetic Bead Panel 2 (Milliplex MAP, EMD Millipore Corp) and CRP was analyzed with the CRPHS Assay (Cobas C, Roche Diagnostics). $\mathrm{PGE}_{2}$ and $\mathrm{TNF}_{\alpha}$ levels were measured twice in the same sample and the mean value was applied, and all other markers were measured in singleton. Further details regarding sample collection are described in the online supplemental methods.

Statistical analyses

For the main analyses, TSPO $\mathrm{V}_{\mathrm{T}}$ was the dependent variable and the natural logarithm of the blood markers $\left(\mathrm{PGE}_{2} / \mathrm{CRP}, \mathrm{PGF}_{2 \mathrm{a}} / \mathrm{CRP}\right.$, and $\mathrm{TNF}_{\mathrm{a}} / \mathrm{CRP}$ ) were each assessed separately as predictor variables in a linear regression in each cohort. The natural logarithm was applied to produce a normal distribution of the predictor variables. To address for the effect of the rs6971 genotype on TSPO $V_{T}$, a corrective adjustment was applied to the mixed-affinity binder (MAB) TSPO $V_{T}$ values based on a linear regression assessing the effect of genotype with regional TSPO $V_{T}$ as the dependent variable such that the differential effect of genotype was added to the TSPO $V_{T}$ of the MAB subjects (see the online Supplementary Information for further description). Since $\mathrm{PGE}_{2} / \mathrm{CRP}$ was the primary hypothesized predictor, the threshold for significance for the linear regression was set at 0.05 for each cohort. For the other two main predictor markers being assessed $\left(\mathrm{PGF}_{2 \mathrm{a}} / \mathrm{CRP}\right.$ and $\left.\mathrm{TNF}_{\mathrm{a}} / \mathrm{CRP}\right)$, the threshold for significance of the linear regression was set at $P<0.017$ since inclusion of these two measures resulted in a total of three high priority measures. For statistical analysis, the adjusted $R^{2}$ was assessed and reported.

Also, receiver operator characteristic (ROC) curves were generated to evaluate the performance of consistently predictive biomarkers. For this, TSPO $\mathrm{V}_{\mathrm{T}}$ values from all subjects were treated as dichotomous variables with PFC TSPO $V_{T} \geq 13.5$ for MDE and $T R D$, and dorsal caudate TSPO $V_{T} \geq 9.4$ for OCD (after adjusting MAB TSPO $V_{T}$ as described in the preceding paragraph). These thresholds correspond to $\sim 30 \%$ greater than healthy controls representing $\sim 2$ standard deviation difference. All analyses were performed using IBM SPSS Statistics (version 21).

\section{RESULTS}

Linear regression assessing relationship of blood markers to TSPO $\mathrm{V}_{\mathrm{T}}$

$\operatorname{Ln}\left(\mathrm{PGE}_{2} / \mathrm{CRP}\right)$ and $\ln \left(\mathrm{TNF}_{\mathrm{a}} / \mathrm{CRP}\right)$ were consistent and highly significant correlates of TSPO $\mathrm{V}_{\mathrm{T}}$ for all three cohorts (Fig. 1, Table 2; $\ln \left(\mathrm{PGE}_{2} / \mathrm{CRP}\right)$ : $M D \mathrm{~F}_{1,19}=10.3$ to $11.8, P=0.0030$ to 0.0048 ; TRD
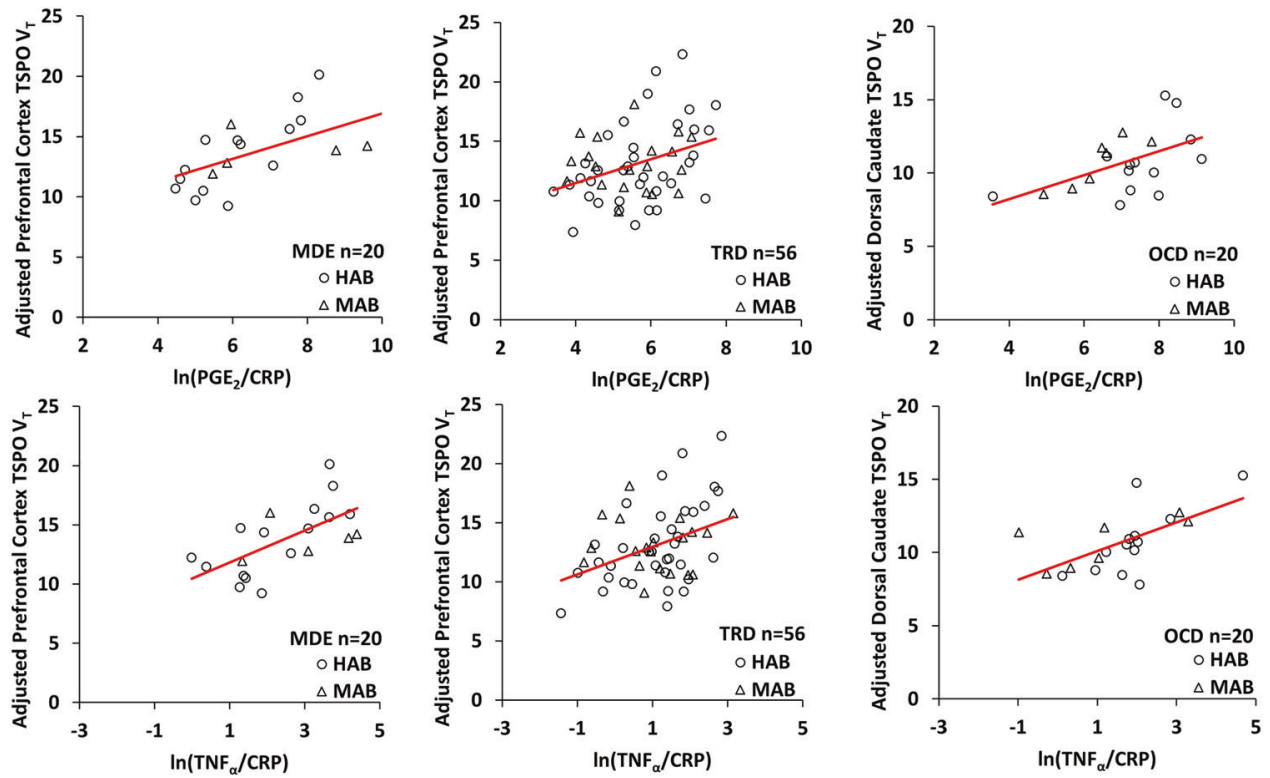

Fig. 1 Blood Serum Markers Significantly Correlated with Translocator Protein Distribution Volume in all Three Cohorts. Ln(PGE $/$ /CRP) and In $\left(\mathrm{TNF}_{\alpha} / \mathrm{CRP}\right)$ were highly significant correlates of PFC TSPO $\mathrm{V}_{\mathrm{T}}$ in MDE and TRD cohorts, and dorsal caudate TSPO $\mathrm{V}_{\mathrm{T}}$ in OCD cohort. MDE cohort $n=20\left[\ln \left(\mathrm{PGE}_{2} / \mathrm{CRP}\right) R^{2}=33.0, P=0.0048 ; \ln \left(\mathrm{TNF}_{\alpha} / \mathrm{CRP}\right) R^{2}=36.2, P=0.0030\right]$. TRD cohort $n=56\left[\ln \left(\mathrm{PGE} \mathrm{E}_{2} / \mathrm{CRP}^{2} R^{2}=10.8, \mathrm{p}=0.0076 ; \ln \left(\mathrm{TNF} \mathrm{F}_{\alpha} /\right.\right.\right.$ CRP) $\left.R^{2}=14.0, P=0.0026\right]$. OCD cohort $n=20\left[\ln \left(\mathrm{PGE}_{2} / \mathrm{CRP}\right) R^{2}=24.1, p=0.016 ; \ln \left(\mathrm{TNF}_{\alpha} / \mathrm{CRP}\right) R^{2}=35.1, P=0.0035\right]$. To address the effect of the rs6971 genotype on TSPO $V_{T}$, the differential effect of genotype in a linear regression was found for TSPO $V_{T}$. Then, the MAB TSPO $V_{T}$ values were adjusted by adding the differential effect of genotype to the TSPO $V_{T}$ values. (Adjusted prefrontal cortex $T S P O V_{T}=u n a d j u s t e d$ TSPO $\mathrm{V}_{\mathrm{T}}+\mathrm{b} 1 *$ genotype, where $\mathrm{b} 1=4.939$ in MDE cohort and $\mathrm{b} 1=4.678$ in TRD cohort. Adjusted dorsal caudate TSPO $\mathrm{V}_{\mathrm{T}}=$ unadjusted TSPO $\mathrm{V}_{\mathrm{T}}+\mathrm{b} 1{ }^{*}$ genotype, where $\mathrm{b} 1=4.230$ in $\mathrm{OCD}$ cohort). CRP c-reactive protein, $H A B$ high-affinity binders, $M A B$ mixed-affinity binders, $M D E$ medication free major depressive episodes secondary to major depressive disorder, OCD obsessive compulsive disorder, PFC prefrontal cortex; $\mathrm{PGE}_{2}$ prostaglandin $\mathrm{E}_{2}, \mathrm{TNF}_{\alpha}$ tumor necrosis factor alpha, TRD treatment resistant major depressive episodes secondary to major depressive disorder, TSPO $V_{\mathrm{T}}$ translocator protein distribution volume 
Table 2. Relationship of serum predictor markers to TSPO $V_{T}$

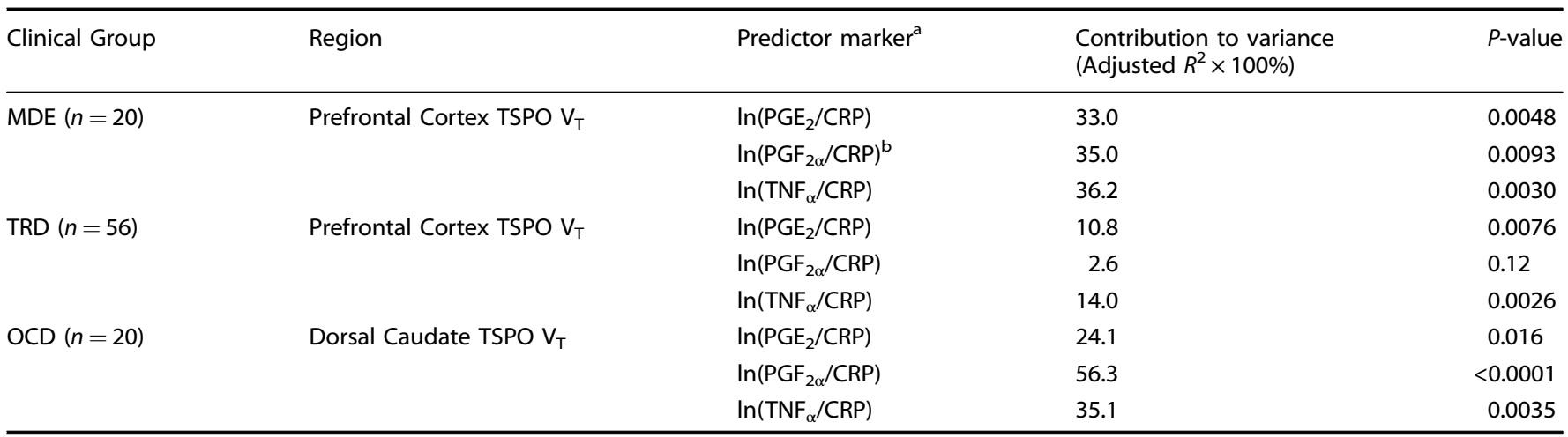

${ }^{a}$ Linear regression with genotyped adjusted TSPO $\mathrm{V}_{\mathrm{T}}$ as dependent variable with predictor listed as independent

${ }^{b} \mathrm{PGF}_{2 \alpha}$ data missing from 4 medication free MDE subjects

$C R P$ c-reactive protein, $M D E$ major depressive episode, $n$ number, $O C D$ obsessive compulsive disorder, $P G E_{2}$ prostaglandin $E 2, P G F_{2 \alpha}$ prostaglandin $F_{2}$ alpha, $T N F_{\alpha}$ tumor necrosis factor alpha, TRD treatment resistant depression, TSPO $V_{T}$ translocator protein distribution volume

A) $\ln \left(\mathrm{PGE}_{2} / \mathrm{CRP}\right)$

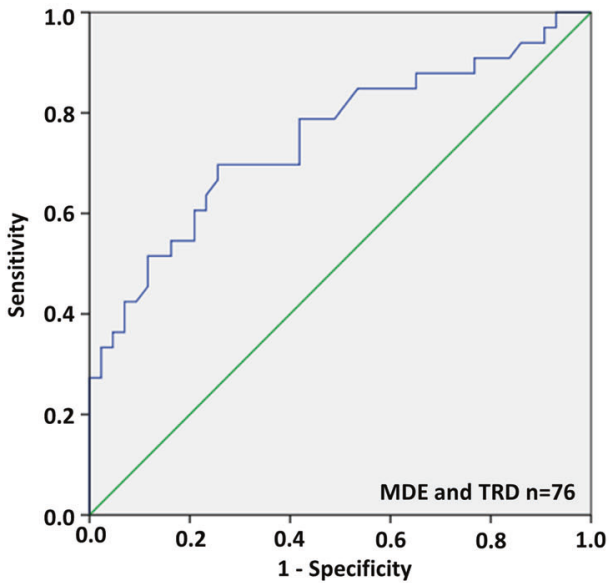

B) $\ln \left(\mathrm{TNF}_{\alpha} / \mathrm{CRP}\right)$

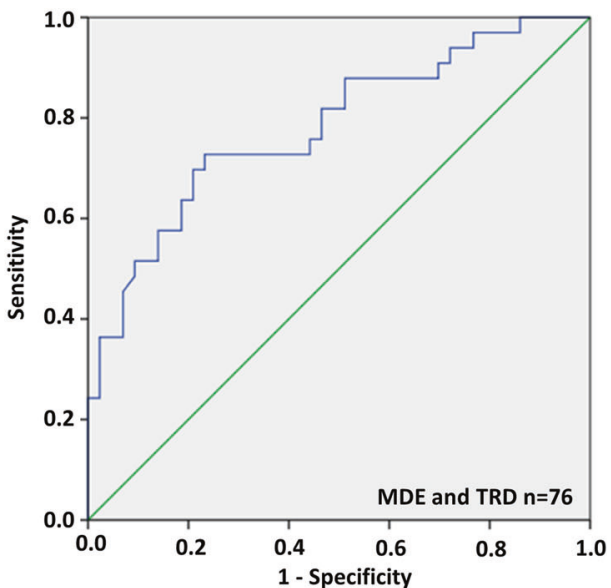

Fig. 2 Receiver Operating Characteristic Curve Analyses in Collective Depressed (MDE and TRD) Cohorts. Receiver operating characteristic (ROC) curve analyses in the collective depressed sample (MDE and TRD $n=76)$ for a) $\ln \left(\mathrm{PGE}_{2} / \mathrm{CRP}\right)$ and $\mathbf{b} \ln \left(\mathrm{TNF} \mathrm{F}_{\alpha} / \mathrm{CRP}\right)$. ROC curve analyses revealed accuracies of $74.9 \%(P=0.00021,95 \%$ confidence interval $[63.5,86.4])$ and $78.1 \%(p<0.0001,95 \%$ confidence interval $[67.5,88.7])$ in the ability of $\ln \left(\mathrm{PGE}_{2} / \mathrm{CRP}\right)$ and $\ln \left(\mathrm{TNF}_{\alpha} / \mathrm{CRP}\right)$ biomarkers to correctly classify those with and without elevated prefrontal cortex TSPO $\mathrm{V}_{\mathrm{T}}$, respectively. To address the effect of the rs6971 genotype on TSPO $V_{T}$, the differential effect of genotype in a linear regression was found for TSPO $V_{T}$. Then, the MAB TSPO $V_{T}$ values were first adjusted by adding the differential effect of genotype to the TSPO $V_{T}$ values. (Adjusted prefrontal cortex TSPO $\mathrm{V}_{\mathrm{T}}=$ unadjusted TSPO $\mathrm{V}_{\mathrm{T}}+\mathrm{b} 1{ }^{*}$ genotype, where $\mathrm{b} 1=4.939$ in MDE cohort and b1 =4.678 in TRD cohort). CRP

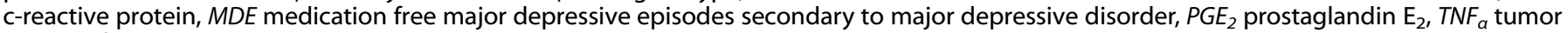
necrosis factor alpha, TRD treatment resistant major depressive episodes secondary to major depressive disorder, TSPO $V_{T}$ translocator protein distribution volume

$\mathrm{F}_{1,55}=7.7$ to $10.0, P=0.0026$ to $0.0076 ; \mathrm{OCD}, \mathrm{F}_{1,19}=7.0$ to 11.3 , $p=0.0035$ to 0.016 ), especially in the MDE and OCD cohorts in which they accounted for 24.1 to $36.2 \%$ of the variance. $\operatorname{Ln}\left(\mathrm{PGF}_{2 \mathrm{a}}\right.$ / CRP) was only significantly predictive in the MDE and OCD cohorts (Table 2; $\mathrm{MDE}, \mathrm{F}_{1,19}=9.1, P=0.0093 ; \mathrm{OCD}, \mathrm{F}_{1,19}=25.5, P<0.0001$ ); accounting for 35.0 and $56.3 \%$ of the variance respectively (see Figure $\mathrm{S} 1$ in the online Supplementary Information). Since TSPO $\mathrm{V}_{\mathrm{T}}$ tended to be highly correlated across regions, the predictors tended to also be correlated with TSPO $\mathrm{V}_{\mathrm{T}}$ values in other brain regions (see Table S2 in the online Supplementary Information).

Receiver operating characteristic curve analysis with A priori hypothesized blood markers

Receiver operating characteristic (ROC) curve analyses were applied for the collective depressed sample (MDE and TRD $n=76$ ) for $\ln \left(\mathrm{PGE}_{2} / \mathrm{CRP}\right)$ and $\ln \left(\mathrm{TNF}_{\mathrm{a}} / \mathrm{CRP}\right)$, assessing revealed an accuracy of
$74.9 \%(P=0.00021,95 \%$ confidence interval $[63.5,86.4])$ and $78.1 \%$ $(P<0.0001,95 \%$ confidence interval $[67.5,88.7])$ in the ability of the blood marker to correctly classify those with and without elevated PFC TSPO $\mathrm{V}_{\mathrm{T}}$ (Fig. 2). ROC curve analyses were applied for the OCD cohort for $\ln \left(\mathrm{PGE}_{2} / \mathrm{CRP}\right), \ln \left(\mathrm{PGF}_{2 \mathrm{a}} / \mathrm{CRP}\right)$, and $\ln \left(\mathrm{TNF}_{\mathrm{a}} /\right.$ CRP), assessing revealed an accuracy of $80.2 \%(P=0.029,95 \%$ confidence interval $[58.4,100.0]), 79.1 \%(P=0.036,95 \%$ confidence interval $[57.5,100.0])$, and $81.3 \%(P=0.024,95 \%$ confidence interval $[60.0,100.0])$, respectively, in the ability of the blood marker to correctly classify those with and without elevated dorsal caudate TSPO $\mathrm{V}_{\mathrm{T}}$ (see Table S3 in the online Supplementary Information). Although the a priori region is different in $O C D$, ROC curve analyses were applied for the whole sample $(n=96)$ to assess the ability of the blood markers to correctly classify those with and without elevated PFC TSPO $V_{T}$ (see Fig. S8 in the online Supplementary Information). 
Relationship of positive predictive value and sample retention In the collective MDE and TRD sample, varying thresholds of In $\left(\mathrm{PGE}_{2} / \mathrm{CRP}\right)$ and $\ln \left(\mathrm{TNF}_{\mathrm{a}} / \mathrm{CRP}\right)$ were assessed in relation to the positive predictive value and proportion of participants retained as these are key practical values for practical use of blood markers in clinical trials. With cut-offs of $\ln \left(\mathrm{PGE}_{2} / \mathrm{CRP}\right)=6.9$ and $\ln \left(\mathrm{TNF}_{\mathrm{a}} /\right.$ $(\mathrm{CRP})=2.1$ respectively in the depressed sample, positive predictive values greater than $80 \%$ for elevated prefrontal cortex TSPO $V_{\mathrm{T}}$ were possible while retaining $40 \%$ of the participants (Fig. 3). Similarly, a threshold serum value of 7.3 for $\ln \left(\mathrm{PGE}_{2} / \mathrm{CRP}\right)$ or 1.9 for $\ln \left(\mathrm{TNF}_{\mathrm{a}} / \mathrm{CRP}\right)$ in OCD subjects selects $\sim 50 \%$ of cases of which $85 \%$ have dorsal caudate TSPO $V_{\mathrm{T}}$ values $>9.4$.

\section{Post-Hoc Analyses}

Since the $\ln \left(\mathrm{PGE}_{2} / \mathrm{CRP}\right)$ or $\ln \left(\mathrm{TNF}_{\mathrm{a}} / \mathrm{CRP}\right)$ may be equivalently expressed as $\ln \left(\mathrm{PGE}_{2}\right)$ minus $\ln (\mathrm{CRP})$ or $\ln \left(\mathrm{TNF}_{\mathrm{a}}\right)$ minus $\ln (\mathrm{CRP})$ respectively, it is feasible to test different linear combinations of $\ln \left(\mathrm{PGE}_{2}\right)$ and $\ln \left(\mathrm{TNF}_{\mathrm{a}}\right)$ with $\ln (\mathrm{CRP})$ with linear regression (see Table S4 in the online Supplementary Information). The most optimal predictor was $\ln (\mathrm{CRP})$ alone which resulted in higher levels of significance in predicting PFC TSPO $V_{T}$ in MDE and TRD participants and dorsal caudate TSPO $V_{\mathrm{T}}$ in OCD participants (MDE: $F_{1,19}=13.0, R^{2}=0.39, P=0.0020$; TRD: $F_{1,55}=17.0, R^{2}=$ $0.23, P=0.00013$; OCD: $\left.F_{1,19}=9.8, R^{2}=0.32, P=0.00058\right)$. The ROC curve analysis of $\operatorname{In}(C R P)$ in the OCD cohort revealed an accuracy of $81.3 \%(P=0.024,95 \%$ confidence interval [61.5, 100.0]) (see Table S3 in the online Supplementary Information). Interestingly, predictiveness increased further in the collective depressed sample (MDE and TRD $n=76$ ) when body mass index (BMI) was included, leading to a ROC curve accuracy of $85.3 \%$ (see the online Supplementary Information for more detail and Figures S2-S7).

\section{DISCUSSION}

We found that composite blood serum measures of products previously established to be synthesized by activated microglia (and to some extent astroglia), that are also known to have strong efflux from the central nervous system, controlled for by peripheral inflammation are consistently significantly correlated with TSPO $\mathrm{V}_{\mathrm{T}}$. The predictiveness of two such measures created a priori, $\operatorname{In}\left(\mathrm{PGE}_{2} / \mathrm{CRP}\right)$ and $\operatorname{In}\left(\mathrm{TNF}_{\mathrm{a}} / \mathrm{CRP}\right)$, replicate across three cohorts. However, it was also noted that $\ln \left(\mathrm{PGF}_{2 \mathrm{a}} / \mathrm{CRP}\right)$ accounted for a high proportion of variance of TSPO $V_{T}$ in the OCD sample and that in post-hoc analyses that the $\ln (\mathrm{CRP})$ was, on average, a stronger predictor of TSPO $\mathrm{V}_{\mathrm{T}}$ across the three cohorts. Low cost predictive markers of TSPO $V_{T}$ have major implications for subject stratification for clinical trials of therapeutics targeting activated microglia.

The present study demonstrates that either serum $\ln \left(\mathrm{PGE}_{2} / \mathrm{CRP}\right)$ or $\ln \left(\mathrm{TNF}_{\mathrm{a}} / \mathrm{CRP}\right)$ consistently identifies a subsample with a high TSPO $V_{T}$ phenotype. Figure 3 , indicates that a threshold serum value of 6.9 for $\ln \left(\mathrm{PGE}_{2} / \mathrm{CRP}\right)$ or 2.1 for $\ln \left(\mathrm{TNF}_{\mathrm{a}} / \mathrm{CRP}\right)$ in the combined MDE and TRD samples selects a subsample of $\sim 40 \%$ of cases of which $80 \%$ have PFC TSPO $\mathrm{V}_{\mathrm{T}}$ values more than 13.5 , which corresponds to 2 standard deviations above the mean of health. Similarly, a threshold serum value of 7.3 for $\ln \left(\mathrm{PGE}_{2} / \mathrm{CRP}\right)$ or 1.9 for $\ln \left(\mathrm{TNF}_{\mathrm{\alpha}} / \mathrm{CRP}\right)$ in OCD subjects $(n=20)$ selects $\sim 50 \%$ of cases of which $85 \%$ have dorsal caudate TSPO $V_{T}$ values $>9.4$. Since the heterogeneity of TSPO $V_{\mathrm{T}}$ elevation ranges from $0 \%$ to over $100 \%$ in primary regions of interest for MDE and OCD [2-4], these serum thresholds represent a practical low-cost option to stratify subjects. Moreover, there will be opportunities to assess these thresholds in clinical trials, particularly for MDD and TRD. For example, therapeutic development to target microglial activation and/or gliosis is actively occurring with P2X7 antagonists for microglial proliferation, and indolamine 2,3 dioxygenase inhibitors to promote conversion of tryptophan towards serotonin rather than the kyurenine pathway. In addition, repurposed medications like minocycline and the simvastatin that influence major histocompatibility protein II to reduce adverse functions of gliosis $[34,35]$ are currently being investigated.

A consistent, positive relationship between blood markers of inflammation and TSPO $V_{T}$ in brain has not been previously reported. In a sample of 30 subjects (14 cases with schizophrenia and 16 healthy), no significant relationships were found between TSPO $\mathrm{V}_{\mathrm{T}}$ and plasma IL-6, $\mathrm{TNF}_{\alpha}$, plasma interferon gamma (IFN $\mathrm{Y}$ ), plasma IL-10, or cerebrospinal fluid IL-6 levels [36]. In a sample of 8 healthy subjects exposed to lipopolysaccharide, Sandiego et al. demonstrated elevations in TSPO $V_{\mathrm{T}}$ as well as $\mathrm{TNF}_{\mathrm{a}}$, plasma IFN $\gamma$, IL-6, IL-8, and IL-10, but there was no correlation between change among brain and peripheral blood measures [37]. Similarly no relationship between blood CRP level and TSPO $V_{T}$ was reported in a sample of $10 \mathrm{MDD}$ subjects some of whom were currently in a MDE, nor in our previous sample of $20 \mathrm{MDE}$ subjects [7, 38]. In a sample of 48 MDD subjects with concurrent TSPO imaging and plasma samples, among 8 plasma measures including CRP, Richards et al. reported one positive correlation of plasma
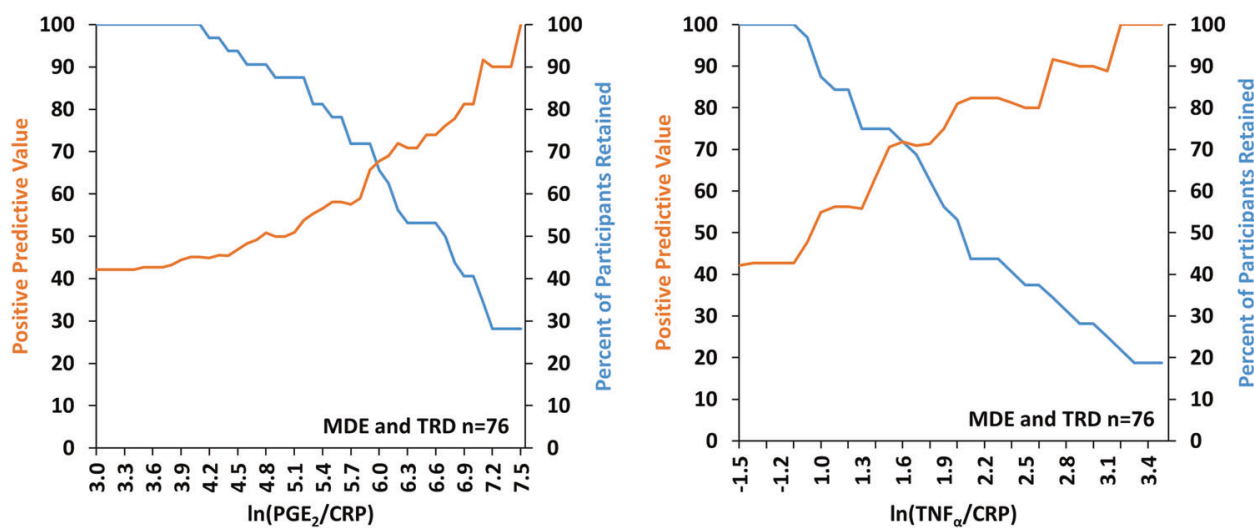

Fig. 3 Relationship of Positive Predictive Value and Proportion of Participants Retained in Collective Depressed (MDE and TRD) Cohorts. Relationship of positive predictive value and proportion of participants retained with varying thresholds of $\ln \left(\mathrm{PGE} \mathrm{E}_{2} / \mathrm{CRP}\right)$ and $\ln \left(\mathrm{TNF}_{\alpha} / \mathrm{CRP}\right)$ in the collective depressed sample (MDE and TRD $n=76$ ). Cut-offs of $\ln \left(\mathrm{PGE}_{2} / \mathrm{CRP}\right)=6.9$ and $\ln \left(\mathrm{TNF}_{\alpha} / \mathrm{CRP}\right)=2.1$ reveal positive predictive values of $\sim 80 \%$ for prefrontal cortex TSPO $\mathrm{V}_{\mathrm{T}} \geq 13.5$ while retaining $40 \%$ of cases. To address the effect of the rs6971 genotype on TSPO $\mathrm{V}_{\mathrm{T}}$, the differential effect of genotype in a linear regression was found for TSPO $V_{T}$. Then, the MAB TSPO $V_{T}$ values were first adjusted by adding the differential effect of genotype to the TSPO $\mathrm{V}_{\mathrm{T}}$ values. (Adjusted prefrontal cortex TSPO $\mathrm{V}_{\mathrm{T}}=$ unadjusted TSPO $\mathrm{V}_{\mathrm{T}}+\mathrm{b} 1{ }^{*} \mathrm{genotype}$, where $\mathrm{b} 1=$ 4.939 in MDE cohort and $\mathrm{b} 1=4.678$ in TRD cohort). CRP c-reactive protein, $P G E_{2}$ prostaglandin $E_{2}, T N F_{\alpha}$ tumor necrosis factor alpha, $T S P O V_{T}$ translocator protein distribution volume 
adiponectin, at an exploratory uncorrected significance [4]. As compared to the present study, there were a number of differences in the study by Richards et al. which examined several different peripheral inflammatory markers (IL-2, IL-5, IL-6, IL-8, and INF- $\gamma$ ) and did not look at the In transformation of CRP in relation to TSPO $\mathrm{V}_{\mathrm{T}}$.

There were several additional predictors that merit further study, due to the high level of variance in TSPO $\mathrm{V}_{\mathrm{T}}$ accounted for by the markers. Although all our cohorts included those with neuropsychiatric disease, the diseases were not identical. Hence, In $\left(\mathrm{PGF}_{2 \mathrm{a}} / \mathrm{CRP}\right)$ which accounted for $56 \%$ of the variance in TSPO $\mathrm{V}_{\mathrm{T}}$ in the dorsal caudate in medication free OCD but was not predictive in the TRD sample, should be reassessed in future samples of medication free OCD subjects since this finding may be specific for OCD. Similarly, since $\ln (\mathrm{CRP})$ and BMI collectively, albeit with most of the impact from In(CRP), accounted for $32 \%$ of the variance and $85.3 \%$ of the area under the ROC to predict elevated TSPO $V_{T}$ in the collective MDE and TRD cohorts; and that the relationship between $\mathrm{BMI}$ and TSPO $\mathrm{V}_{\mathrm{T}}$ was also reported in a separate sample from ours [4] suggest that this combination should receive further study in MDE and TRD samples.

There are several limitations in the present study. First, there is some lack of selectivity for gliosis across the markers tested. For example, when elevated TSPO $V_{T}$ is present, it is associated with microglial activation but TSPO overexpression is not fully selective for microglial activation since TSPO is detectable in other cells such as astroglia and endothelial cells $[11,12]$. Also, some of the serum markers measured like $\mathrm{PGE}_{2}, \mathrm{PGF}_{2 a}$ and $\mathrm{TNF}_{\mathrm{a}}$, while synthesized by activated microglia are not specific to such, for example, they may also be produced by activated astrocytes $[39,40]$. Second, when greater TSPO expression often occurs after exposure to inflammatory stimuli, it is associated with the morphological changes of an activated state, predominantly in microglia but there is a range of cellular functions that may occur in the activated state. Third, we did not assess the relationship of the prioritized blood markers to elevated brain TSPO $V_{\mathrm{T}}$ in healthy subjects because in health, TSPO $V_{T}$ has a limited range. In addition, in neuroinflammatory states the elevation in TSPO $V_{T}$ is generally viewed as reflecting variable levels of gliosis whereas the range in health is likely attributable to variation in other aspects of TSPO binding like binding to endothelial cells [10]. Fourth, it could be questioned as to which peripheral blood markers were decided a priori, a challenging issue since disclosing these prior to patenting in a public database invalidates the patent and then limits the translational impact of such markers. We can largely address this question by our emerging patenting order of peripheral blood markers which demonstrates initial selection of $\mathrm{PGE}_{2} / \mathrm{CRP}$ and later selection of our post-hoc parameter $\ln (\mathrm{CRP})$, which in some examples both alone and in combination with $B M I$ value yields even greater levels of predictiveness for TSPO $V_{T}$ (see Figures S5-S7 in the online Supplementary Information).

In addition, should the serum markers of the present study be applied to other studies, it is important that the sampling protocol is highly similar to ours. One important component of our sampling protocol is that to avoid potentially rapid degradation of $\mathrm{PGE}_{2}$ and $\mathrm{TNF}_{\mathrm{a}}$, blood samples taken were converted into serum over $30 \mathrm{~min}$, placed in a chilled centrifuge, and stored quickly at $-80^{\circ} \mathrm{C}$ (see the online Supplementary Information). Also, to apply the relationship of the serum measures tested to TSPO $V_{T}$, it is also important to address whether participants sampled had recent infections since our participant sample reported no recent infections in the four weeks prior to scanning which may have lowered variability in the TSPO $V_{T}$ measure.

In summary, we assessed a general strategy of testing the serum ratio of products synthesized by activated microglia that are also actively removed from the central nervous system, controlled for by markers of peripheral inflammation, identified the natural logarithm of serum $\mathrm{PGE}_{2} / \mathrm{CRP}$ and $\mathrm{TNF}_{\alpha} / \mathrm{CRP}$, as highly significant correlates of TSPO $\mathrm{V}_{\mathrm{T}}$ in brain in three separate samples. We also noted in post-hoc analyses several linear combinations of markers that were also highly predictive including $\ln (\mathrm{CRP})$ itself as well as in combination with BMI. These measures may be applied at thresholds with high positive predictive value to select subjects with elevated TSPO $V_{T}$ thereby addressing the heterogeneity of the TSPO $V_{T}$ marker when recruiting subjects for clinical trials of therapeutics targeting gliosis. Moreover, systematically assessing collective predictors in relation to TSPO $V_{T}$ has intriguing potential to be developed further towards more individualized clinical care of neuropsychiatric illnesses with elevated microglial activation and gliosis.

\section{FUNDING AND DISCLOSURES}

This study received funding support from the Canadian Institutes of Health Research [Canada Research Chair and Operating Grant (MOP136955), JHM; Doctoral Award (GSD-157948), SA; Fellowship Award, ES; Canada Research Chair, NV], the Brain and Behavior Foundation, and the neuroscience catalyst fund (from the Government of Ontario and Janssen). None of the funding sources participated in the execution of the project, generation of results, interpretation of data nor drafting of the manuscript. Funding for infrastructure was from the Azrieli Foundation, the Canadian Foundation for Innovation and the Ontario Ministry for Innovation. All other authors are paid employees of the Centre for Addiction and Mental Health. AAW, SH, and JHM have received operating grant funds from Janssen in the past 5 years. JHM has been a consultant to Lundbeck and Takeda, in the past 5 years. JHM is an inventor on five patents of blood and/or clinical markers to predict brain inflammation or to diagnose affective disorders (including those in the present submission), and a dietary supplement to reduce depressed mood post-partum. JHM is arranging collaborations with nutraceutical companies for the dietary supplement to prevent post-partum depression. SK has operating grant funds from US National Institutes of Health National Institute on Drug Abuse (DA04066) to measure microglial status in the brains of methamphetamine users, and from Jazz Pharmaceuticals for an unrelated study.

\section{ACKNOWLEDGEMENTS}

AN, LN, and CC worked as study PET technicians. AK, NK, AS, and RM provided medical coverage for the PET scans. JP, AG, and Michael Harkness of Research Imaging Centre, served as PET chemistry staff. AR, GED, and HB, worked as study MRI technicians. All other contributors are paid employees of the Centre for Addiction and Mental Health.

\section{AUTHOR CONTRIBUTIONS}

Study concept and design: JHM, ES, AAW, SH. Acquisition, analysis, or interpretation of data: SA, ES, PMR, LM, CX, CH, SK, JHM. Drafting of the manuscript: SA, JHM. Critical revision of the manuscript for important intellectual content: $S A$, Setiawan, Wilson, PMR, LM, CX, CH, MIH, SK, NV, SH, JHM. Statistical analysis: SA, JHM. Obtained funding: SA, JHM, ES, AAW, SH. Administrative, technical, or material support: AAW, PMR, LM, SK, NV, SH, JHM. Study supervision: JHM.

\section{ADDITIONAL INFORMATION}

Supplementary Information accompanies this paper at (https://doi.org/10.1038/ s41386-019-0561-y).

Publisher's note Springer Nature remains neutral with regard to jurisdictional claims in published maps and institutional affiliations.

\section{REFERENCES}

1. WHO. 169 (World Health Organization, Geneva, Switzerland, 2001).

2. Attwells S, Setiawan E, Wilson AA, Rusjan PM, Mizrahi R, Miler L, et al. Inflammation in the neurocircuitry of obsessive-compulsive disorder. JAMA Psychiatry. 2017;74:833-40. 
3. Setiawan E, Attwells S, Wilson AA, Mizrahi R, Rusjan PM, Miler L, et al. Association of translocator protein total distribution volume with duration of untreated major depressive disorder: a cross-sectional study. Lancet Psychiatry. 2018;5:339-47.

4. Richards EM, Zanotti-Fregonara P, Fujita M, Newman L, Farmer C, Ballard ED, et al $\mathrm{PET}$ radioligand binding to translocator protein (TSPO) is increased in unmedicated depressed subjects. EJNMMI Res. 2018;8:57.

5. Li H, Sagar AP, Keri S. Translocator protein (18kDa TSPO) binding, a marker of microglia, is reduced in major depression during cognitive-behavioral therapy. Prog neuro-Psychopharmacol Biol Psychiatry 2018;83:1-7.

6. Hickman S, Izzy S, Sen P, Morsett L, El Khoury J. Microglia in neurodegeneration. Nat Neurosci 2018;21:1359-69.

7. Setiawan E, Wilson AA, Mizrahi R, Rusjan PM, Miler L, Rajkowska G, et al. Role of translocator protein density, a marker of neuroinflammation, in the brain during major depressive episodes. JAMA Psychiatry. 2015;72:268-75.

8. Holmes SE, Hinz R, Conen S, Gregory CJ, Matthews JC, Anton-Rodriguez JM, et al. Elevated translocator protein in anterior cingulate in major depression and a role for inflammation in suicidal thinking: a positron emission tomography study. Biol Psychiatry 2018;83:61-9.

9. Block ML, Zecca L, Hong JS. Microglia-mediated neurotoxicity: uncovering the molecular mechanisms. Nat Rev Neurosci. 2007;8:57-69.

10. Betlazar C, Harrison-Brown M, Middleton RJ, Banati R, Liu GJ. Cellular sources and regional variations in the expression of the neuroinflammatory marker translocator protein (TSPO) in the normal brain. Int J Mol Sci. 2018;19:pii: E2707.

11. Banati RB, Myers R, Kreutzberg GW. PK ('peripheral benzodiazepine')-binding sites in the CNS indicate early and discrete brain lesions: microautoradiographic detection of [3H]PK11195 binding to activated microglia. J Neurocytol. 1997;26:77-82.

12. Martin A, Boisgard R, Theze B, Van Camp N, Kuhnast B, Damont A, et al. Evaluation of the PBR/TSPO radioligand [(18)F]DPA-714 in a rat model of focal cerebral ischemia. J Cereb Blood Flow Metab: Off J Int Soc Cereb Blood Flow Metab. 2010;30:230-41.

13. Narayanaswami V, Dahl K, Bernard-Gauthier V, Josephson L, Cumming $P$, Vasdev N. Emerging PET radiotracers and targets for imaging of neuroinflammation in neurodegenerative diseases: outlook beyond TSPO. Mol imaging 2018;17:1536012118792317.

14. Horti AG, Naik R, Foss CA, Minn I, Misheneva V, Du Y, et al. PET imaging of microglia by targeting macrophage colony-stimulating factor 1 receptor (CSF1R). Proceedings of the National Academy of Sciences of the United States of America. 2019.

15. Galasko D, Montine TJ. Biomarkers of oxidative damage and inflammation in Alzheimer's disease. Biomark Med. 2010;4:27-36.

16. Wang AK, Miller BJ. Meta-analysis of cerebrospinal fluid cytokine and tryptophan catabolite alterations in psychiatric patients: comparisons between schizophrenia, bipolar disorder, and depression. Schizophrenia Bull 2018;44:75-83.

17. Pulli B, Chen JW. Imaging neuroinflammation - from bench to bedside. J Clin Cell Immunol. 2014;5:pii: 226.

18. Minghetti L, Polazzi E, Nicolini A, Creminon C, Levi G. Up-regulation of cyclooxygenase-2 expression in cultured microglia by prostaglandin E2, cyclic AMP and non-steroidal anti-inflammatory drugs. Eur J Neurosci. 1997;9:934-40.

19. Akanuma S, Higuchi T, Higashi H, Ozeki G, Tachikawa $M$, Kubo $Y$, et al. Transporter-mediated prostaglandin $\mathrm{E}(2)$ elimination across the rat blood-brain barrier and its attenuation by the activation of $\mathrm{N}$-methyl-D-aspartate receptors. Drug Metab pharmacokinetics. 2014;29:387-93.

20. Tachikawa M, Hosoya K, Terasaki T. Pharmacological significance of prostaglandin E2 and D2 transport at the brain barriers. Adv Pharm. 2014;71:337-60.
21. Banks WA. The blood-brain barrier in neuroimmunology: tales of separation and assimilation. Brain Behav Immun. 2015;44:1-8

22. Hagen AA, Gerber JN, Sweeley CC, White RP, Robertson JT. Levels and disappearance of prostaglandin F2alpha in cerebral spinal fluid: a clinical and experimental study. Stroke 1977;8:672-5.

23. Kalinski P. Regulation of immune responses by prostaglandin E2. J Immunol 2012;188:21-8.

24. Ressler KJ, Mayberg HS. Targeting abnormal neural circuits in mood and anxiety disorders: from the laboratory to the clinic. Nat Neurosci 2007;10:1116-24.

25. Drevets WC, Price JL, Furey ML. Brain structural and functional abnormalities in mood disorders: implications for neurocircuitry models of depression. Brain Struct Funct. 2008;213:93-118.

26. Williams LM. Precision psychiatry: a neural circuit taxonomy for depression and anxiety. Lancet Psychiatry. 2016;3:472-80.

27. Adams KH, Hansen ES, Pinborg LH, Hasselbalch SG, Svarer C, Holm S, et al. Patients with obsessive-compulsive disorder have increased 5-HT2A receptor binding in the caudate nuclei. Int J Neuropsychopharmacol. 2005;8:391-401.

28. Wong DF, Brasic JR, Singer HS, Schretlen DJ, Kuwabara H, Zhou Y, et al. Mechanisms of dopaminergic and serotonergic neurotransmission in Tourette syndrome: clues from an in vivo neurochemistry study with PET. Neuropsychopharmacology. 2008;33:1239-51.

29. Saxena S, Brody AL, Schwartz JM, Baxter LR. Neuroimaging and frontal-subcortical circuitry in obsessive-compulsive disorder. Br. J. Psychiatry. 1998;35:26-37.

30. Hamilton M. A rating scale for depression. J Neurol Neurosurg Psychiatry. 1960; 23:56-62.

31. Goodman WK, Price LH, Rasmussen SA, Mazure C, Delgado P, Heninger GR, et al The yale-brown obsessive compulsive scale. II. Validity. Arch Gen Psychiatry. 1989;46:1012-6.

32. Rusjan P, Mamo D, Ginovart N, Hussey D, Vitcu I, Yasuno F, et al. An automated method for the extraction of regional data from PET images. Psychiatry Res. 2006;147:79-89.

33. Rusjan PM, Wilson AA, Bloomfield PM, Vitcu I, Meyer JH, Houle S, et al. Quantitation of translocator protein binding in human brain with the novel radioligand [18F]-FEPPA and positron emission tomography. J Cereb Blood Flow Metab. 2011; 31:1807-16.

34. Kwak B, Mulhaupt F, Myit S, Mach F. Statins as a newly recognized type of immunomodulator. Nat Med. 2000;6:1399-402.

35. Henry CJ, Huang Y, Wynne A, Hanke M, Himler J, Bailey MT, et al. Minocycline attenuates lipopolysaccharide (LPS)-induced neuroinflammation, sickness behavior, and anhedonia. J Neuroinflammation. 2008;5:15.

36. Coughlin JM, Wang Y, Ambinder EB, Ward RE, Minn I, Vranesic M, et al. In vivo markers of inflammatory response in recent-onset schizophrenia: a combined study using [(11)C]DPA-713 PET and analysis of CSF and plasma. Transl Psychiatry. 2016;6:e777.

37. Sandiego CM, Gallezot JD, Pittman B, Nabulsi N, Lim K, Lin SF, et al. Imaging robust microglial activation after lipopolysaccharide administration in humans with PET. Proc Natl Acad Sci USA. 2015;112:12468-73.

38. Hannestad J, DellaGioia N, Gallezot JD, Lim K, Nabulsi N, Esterlis I, et al. The neuroinflammation marker translocator protein is not elevated in individuals with mild-to-moderate depression: a [(1)(1)C]PBR28 PET study. Brain Behav Immun. 2013:33:131-8

39. Saito O, Svensson Cl, Buczynski MW, Wegner K, Hua XY, Codeluppi S, et al. Spinal glial TLR4-mediated nociception and production of prostaglandin $\mathrm{E}(2)$ and TNF. Br J Pharmacol. 2010;160:1754-64.

40. Dong Y, Benveniste EN. Immune function of astrocytes. Glia 2001;36:180-90. 